\title{
ON g-CONCAVE FUNCTIONS AND PROBABILITY MEASURES
}

The notions of convexity and concavity play an important role in many aspects of mathematical programming. To extend the classes of convex and concave functions, various concepts of generalized convexity and concavity have been introduced and investigated by several authors, e. g., $\left[{ }^{1-7}\right]$. In particular, Avriel and Zang $\left[{ }^{1}\right]$ introduced the notion of $G$-concave function. In the present paper the concept of $G$-concavity is, in a slightly modified form, applied to probability measures and investigated from the stochastic programming point of view. The results generalize those of Prékopa $\left[{ }^{2}\right]$.

Let $g: G_{1} \rightarrow G_{2}$, where $G_{1}, G_{2} \subseteq R^{1}$, be a strictly monotone increasing function.

Definition 1. A function $f: F_{1} \rightarrow F_{2}$, where $F_{1} \subseteq R^{n}$ is a convex set and $F_{2} \subseteq G_{1}$, is called $g$-concave (g-convex) on $F_{1}$ if $g(f(x))$ is concave (convex) on $F_{1}$.

In other words, $f(x)$ is $g$-concave on $F_{1}$ if

$$
g\left(f\left(\lambda x_{1}+(1-\lambda) x_{2}\right)\right) \geqslant \lambda g\left(f\left(x_{1}\right)\right)+(1-\lambda) g\left(f\left(x_{2}\right)\right)
$$

and g-convex if

$$
g\left(f\left(\lambda x_{1}+(1-\lambda) x_{2}\right)\right) \leqslant \lambda g\left(f\left(x_{1}\right)\right)+(1-\lambda) g\left(f\left(x_{2}\right)\right)
$$

for all $x_{1}, x_{2} \in F_{1}$ and $\lambda \in[0,1]$.

It is obvious that if $f(x)$ is $g$-convex on some set $F$ then $-f(x)$ is $g$-concave on this set.

The concept of $g$-concavity differs from that of $G$-concavity introduced by Avriel and Zang $\left[{ }^{1}\right]$ only by the fact that $g$ is a strictly monotone increasing function, whereas $G$ is supposed to be only strictly monotone.

The logarithmic concavity $\left[{ }^{3}\right]$ is a particular case of the $g$-concavity. In this case $g(t)=\ln t$. On the other hand, there are functions which are not logarithmic concave but are $g$-concave. For example, $f(x)=x^{3}$, $x \in R^{1}$ is negative on the set and hence it cannot be logarithmic concave but it is $g$-concave if $g(t)=\sqrt[3]{t}$.

Let us establish some properties of $g$-concave functions.

The or e m 1. A function which is concave on $F_{1}$ is g-concave on $F_{1}$ with respect to every $g(t)$, concave on $F_{2}$.

$\mathrm{Proof}$ is based on the fact that a monotone increasing concave function of a concave function is concave.

The or em 2. Let $g(t)$ be a differentiable function on $F_{2}$. If $f(x)$ is differentiable and $g$-concave on $F_{1}$ then it is pseudo-concave* on $F_{1}$.

* A differentiable function $h: F_{1} \rightarrow F_{2}$ is called pseudo-concave on $F_{1}$ if for any $x_{1}, x_{2} \in F_{1} h^{\prime}\left(x_{1}\right)\left(x_{2}-x_{1}\right) \leqslant 0$ implies $h\left(x_{2}\right) \leqslant h\left(x_{1}\right)$. 
Theorem 3. A function which is g-concave on some set is quasiconcave** on that set.

$\mathrm{Pro}$ of $\mathrm{s}$ of theorems 2 and 3 are readily obtained from $\left[{ }^{1}\right]$.

The converses of the theorems 2 and 3 do not hold. One can find pseudo-concave and, all the more, quasi-concave functions $f(x)$ for which no such strictly monotone increasing function $g(t)$ exists that $g(f(x))$ is concave. For example, let us consider the function

$$
\varphi(x)= \begin{cases}-\frac{1}{4} x^{2}+\frac{1-\pi}{4}, & \text { if } x \leqslant 1 \\ -\operatorname{arctg} x, & \text { if } x>1,\end{cases}
$$

where $x \in F_{1}=R^{1}$. It can be easily shown that this function is pseudoconcave. To show that $\varphi(x)$ is not $g$-concave we shall prove the following lemma.

$\mathrm{L}$ e $\mathrm{mma}$. On the set $[a, \infty]$ concave, monotone decreasing and bounded function $h(x)$ is constant on this set.

To prove this statement, let us denote $m=\inf _{x \in[a, \infty)} h(x)$ and $M=$


$\varepsilon>0, \bar{x}$ so that $h(\bar{x})<m+\varepsilon, x_{1}=a$ and $x_{2}$ so that $\bar{x}=\frac{x_{1}+x_{2}}{2}$. Because of the concavity of $h(x)$ we have $m+\varepsilon>h(\bar{x})=h\left(\frac{x_{1}+x_{2}}{2}\right) \geqslant \frac{1}{2} h\left(x_{1}\right)+$ $+\frac{1}{2} h\left(x_{2}\right)=\frac{1}{2} M+\frac{1}{2} h\left(x_{2}\right) \geqslant \frac{1}{2} M+\frac{1}{2} m$. Therefore, $m+\varepsilon>\frac{1}{2}(M+m)$. If we take now $\varepsilon=\frac{M-m}{2}>0$, we come to the contradiction $\frac{m+M}{2}>$ $>\frac{m+M}{2}$ and, hence, $h(x)$ must be constant.

Let us assume now that there exists a strictly monotone increasing function $g(t)$ so that $g(\varphi(x))$ is a concave function. Then $g(t)$ must be defined on the set $\left(-\infty, \frac{1-\pi}{4}\right]$. Therefore $g_{0}=g(\varphi(0))=g\left(\frac{1-\pi}{4}\right)$ and $g_{1}=g(\varphi(+\infty))=g\left(-\frac{\pi}{2}\right)$ are finite. So $g(\varphi(x))$ is a concave monotone decreasing and bounded function on the set $[0, \infty)$. By the lemma, $g(\varphi(x))$ is constant. But this contradicts the condition that $g(t)$ is strictly monotone.

Theorem 4. If $f(x)$ is g-concave on $F_{1}, X \subseteq F_{1}$ is a convex set and $\max _{x \in X} f(x)$ exists then $g\left(\max _{x \in X} f(x)\right)=\max _{x \in X} g(f(x))$.

Proof. Let $\max _{x \in X} f(x)=f\left(x^{*}\right), x^{*} \in X$. Then $f(x) \leqslant f\left(x^{*}\right)$ for every $x \in X$. As $g(t)$ is a strictly increasing function, $g(f(x)) \leqslant g\left(f\left(x^{*}\right)\right)$. Consequently, $\max _{x \in X} g(f(x))=g\left(f\left(x^{*}\right)\right)=g\left(\max _{x \in X} f(x)\right)$.

Theorem 4 can be used to replace the problem $\max _{x \in X} f(x)$ with an equivalent concave problem $\max _{x \in X} g(f(x))$ as soon as $f(x)$ is $g$-concave.

In stochastic programming problems containing probabilistic cost functions or restrictions it is important to have conditions under which these *** A function $h(x)$ is called quasi-concave on a convex set $F_{1} \subseteq R^{n}$ if $h\left(\lambda x_{1}+\right.$ $\left.+(1-\lambda) x_{2}\right) \geqslant \min \left[h\left(x_{1}\right), h\left(x_{2}\right)\right]$ for every $x_{1}, x_{2} \in F_{1}$ and every $\lambda \in[0,1]$. 
functions are $g$-concave. For this purpose we shall introduce the following notion.

Definition 2. A probability measure $P$, defined on a $\sigma$-algebra $\Sigma$ of sets from $R^{m}$, is called g-concave if for every pair of convex sets $A, B \in \Sigma$ and $\lambda \in(0,1)$ the inequality

$$
g\{P[\lambda A+(1-\lambda) B]\} \geqslant \lambda g\{P[A]\}+(1-\lambda) g\{P[B]\}
$$

holds.

This notion is a generalization of the logarithmic concave probability measure considered in $\left[{ }^{2}\right]$.

Theorem 5. If $f(x, y)$ is quasi-concave on $R^{n+m}, x \in R^{n}$ and $y \in R^{m}$ is a random vector corresponding to a g-concave probability measure $P$ then the probabilistic function $v(x)=P[f(x, y) \geqslant 0]$ is $g$ concave.

P r o o f. Let us denote $H(x)=\{y: f(x, y) \geqslant 0\}$. Then

$$
v(x)=P[H(x)] \text {. }
$$

As the function $f(x, y)$ is quasi-concave, so

$$
f\left(\lambda x_{1}+(1-\lambda) x_{2}, \lambda y_{1}+(1-\lambda) y_{2}\right) \geqslant \min \left[f\left(x_{1}, y_{1}\right), f\left(x_{2}, y_{2}\right)\right] \text {. }
$$

Any element of the set $\lambda H\left(x_{1}\right)+(1-\lambda) H\left(x_{2}\right)$ can be expressed in the form $\lambda y_{1}+(1-\lambda) y_{2}$, where $y_{1} \in H\left(x_{1}\right), y_{2} \in H\left(x_{2}\right)$, i. e., $f\left(x_{1}, y_{1}\right) \geqslant 0$ and $f\left(x_{2}, y_{2}\right) \geqslant 0$. Because of $(5) f\left(\lambda x_{1}+(1-\lambda) x_{2}, \lambda y_{1}+(1-\lambda) y_{2}\right) \geqslant 0$, that is $\lambda y_{1}+(1-\lambda) y_{2} \in H\left(\lambda x_{1}+(1-\lambda) x_{2}\right)$. Therefore, $H\left(\lambda x_{1}+(1-\lambda) x_{2}\right) \supseteq$ $\supseteq \lambda H\left(x_{1}\right)+(1-\lambda) H\left(x_{2}\right)$ and $P\left\{H\left(\lambda x_{1}+(1-\lambda) x_{2}\right)\right\} \geqslant P\left\{\lambda H\left(x_{1}\right)+(1-\lambda) \times\right.$ $\left.\times H\left(x_{2}\right)\right\}$. Taking into account the monotonity of $g(t)$ and the $g$-concavity of the measure $P$ we get $g\left\{P\left[H\left(\lambda x_{1}+(1-\lambda) x_{2}\right)\right]\right\} \geqslant g\left\{P\left[\lambda H\left(x_{1}\right)+\right.\right.$ $\left.\left.+(1-\lambda) H\left(x_{2}\right)\right]\right\} \geqslant \lambda g\left\{P\left[H\left(x_{1}\right)\right]\right\}+(1-\lambda) g\left\{P\left[H\left(x_{2}\right)\right]\right\}$, and hence, because of $(4), g\left(v\left(\lambda x_{1}+(1-\lambda) x_{2}\right)\right) \geqslant \lambda g\left(v\left(x_{1}\right)\right)+(1-\lambda) g\left(v\left(x_{2}\right)\right)$.

In conclusion we shall demonstrate that the probability measure corresponding to the one-dimensional Cauchy distribution is not logarithmic concave but it is $g$-concave if $g(t)=\operatorname{tg}[\pi(t-1 / 2)]$.

In one-dimensional case a convex set is a segment, a semi-segment or an interval, i. e., it is determined by a couple $\langle u, v\rangle$, where $u, v$ are either numbers or $+\infty$, or $-\infty$ and $u<v$. If $A=\left\langle u_{1}, v_{1}\right\rangle, B=\left\langle u_{2}, v_{2}\right\rangle$ then $\lambda A+(1-\lambda) B=\left\langle\lambda u_{1}+(1-\lambda) u_{2}, \lambda v_{1}+(1-\lambda) v_{2}\right\rangle$ and $(3)$ takes the form $g\left\{P\left\langle\lambda u_{1}+(1-\lambda) u_{2}, \lambda v_{1}+(1-\lambda) v_{2}\right\rangle\right\} \geqslant \lambda g\left\{P\left\langle u_{1}, v_{1}\right\rangle\right\}+(1-\lambda) g\left\{P\left\langle u_{2}, v_{2}\right\rangle\right\}$ that coincides with the condition of g-concavity of a function of two variables.

Let us show that the function $P\{\langle u, v\rangle\}=\frac{1}{\pi}(\operatorname{arctg} v-\operatorname{arctg} u)$ corresponding to the one-dimensional Cauchy distribution is $g$-concave if $g(t)=\operatorname{tg}[\pi(t-1 / 2)]$ but is not logarithmic concave. To demonstrate it we shall evaluate the matrices of second order derivatives of the functions $\ln [1 / \pi(\operatorname{arctg} v-\operatorname{arctg} u)]$ and $\operatorname{tg}[\pi(1 / \pi(\operatorname{arctg} v-\operatorname{arctg} u)-1 / 2)]$

$$
\frac{1}{(\operatorname{arctg} v-\operatorname{arctg} u)^{2}}\left(\begin{array}{cc}
\frac{-1+2 u(\operatorname{arctg} v-\operatorname{arctg} u)}{\left(1+u^{2}\right)^{2}} & \frac{1}{\left(1+u^{2}\right)\left(1+v^{2}\right)} \\
\frac{1}{\left(1+u^{2}\right)\left(1+v^{2}\right)} & \frac{-1-2 v(\operatorname{arctg} v-\operatorname{arctg} u)}{\left(1+v^{2}\right)^{2}}
\end{array}\right)
$$


and

$$
\frac{2}{(u-v)^{3}}\left(\begin{array}{cc}
1+v^{2} & -u v-1 \\
-u v-1 & 1+u^{2}
\end{array}\right),
$$

respectively.

If $-1+2 u(\operatorname{arctg} v-\operatorname{arctg} u)>0$ (e. g. $u=1, v=4)$ then the element in the first row and first column

$$
\frac{-1+2 u(\operatorname{arctg} v-\operatorname{arctg} u)}{(\operatorname{arctg} v-\operatorname{arctg} u)^{2}\left(1+u^{2}\right)^{2}}>0 .
$$

Consequently, (6) is not a negative semi-definite matrix for every $u, v \in R^{1}$.

But it is easy to see that the matrix (7) is negative semi-definite for every $u, v \in R^{1}$ and hence the function $\operatorname{tg}[\pi(1 / \pi(\operatorname{arctg} v-\operatorname{arctg} u)-1 / 2)]$ is concave.

\section{REFERENCES}

1. Avrie 1, M., Z ang, I., Mathematical Programs for Activity Analysis, Amsterdam, 1974.

2. Pré k o p a, A., Math. Operationsforsch. u. Statist., 3, H. 5, 349 (1972).

3. Klinger, A., Mang a sarian, O. L., J. Math. Analysis and Applic., No. 2, 388 (1968).

4. Avriel, M., Math. Program., No. 2, 309 (1972).

5. Thom pson, Jr., W. A., P a rke, Darrel, W., Operat. Res., 21, No. 1, 305 (1973).

6. Bere a n u, B., R.A.I.R.O., 6, No. 1, 15 (1972).

7. $З$ ан г в иял У. И., Нелинейное пропраммирование, М., 1973.
Academy of Sciences of the Estonian SSR, Institute of Cybernetics
Received
Nov. 23, 1976

Ebu TAMM

\section{g-NOGUSATEST FUNKTS!OONIDEST JA TOENÄOSUSMOOTUDEST}

Defineeritakse $g$-nõgusa funktsiooni ja $g$-nõgusa tõenäosusmõõdu mõisted kui vastavâlt logaritmiliselt nõgusa funktsiooni ja logaritmiliselt nōgusa tõenäosusmõõdu üldistused. Vaadeldakse $g$-nôgusate funktsioonide omadusi ja esitatakse tingimused, millal tōenäosusfunktsioon $v(x)=P[f(x, y) \geqslant 0]$ on $g$-nõgus. Näidatakse, et ühedimensionaalsele Cauchy jaotusele vastav tõenäosusmõõt on g-nōgus, kuid ei ole logaritmiliselt nõgus.

\section{Эбу $T A M M$}

\section{O g-вОГНУТЫХ ФУНКЦИЯХ И ВЕРОятНОСТНЫХ МЕРАХ}

Определяются понятия $g$-вогнутой функции и $g$-вогнутой вероятностной меры как обобщения логарифмически вопнутой функции и логарифмически вопнутой вероятностной меры соответственно. Рассматриваются некоторые свойства $g$-вопнутых функций и даются условия, при которых функция вероятности $v(x)=P[f(x, y) \geqslant 0]$ $g$-вопнута. Показывается, что вероятностная мера, соответствующая одномерному распределению Коши, g-вогнута, но логарифмически невогнута, 